Article

\title{
Integrating Spatial and Non-Spatial Dimensions to Measure Urban Fire Service Access
}

\author{
Zelong Xia ${ }^{1,2}$, Hao Li ${ }^{1, *}$, Yuehong Chen ${ }^{1}$ and Wenhao $\mathrm{Yu}^{3}$ \\ 1 School of Earth Sciences and Engineering, Hohai University, Nanjing 210098, China; \\ 160211020003@hhu.edu.cn (Z.X.); yuehongchen@hhu.edu.cn (Y.C.) \\ 2 Beijing Key Laboratory of Urban Spatial Information Engineering, Beijing 100089, China \\ 3 Faculty of Information Engineering, China University of Geosciences, Wuhan 430074, China; \\ yuwh@cug.edu.cn \\ * Correspondence: lihao@hhu.edu.cn; Tel.: +86-136-0515-4865
}

Received: 7 February 2019; Accepted: 11 March 2019; Published: 13 March 2019

\begin{abstract}
Assessing the access to fire service at an urban scale involves accounting for geographical impedance, demand, and supply, thus both spatial and non-spatial dimensions must be taken into account. Therefore, in this paper, an optimized two-step floating catchment area (F-2SFCA) method is proposed for measuring urban fire service access, which incorporates the effects of both spatial and non-spatial factors into fire service access. The proposed model is conducted in a case study to assess the fire service accessibility of Nanjing City, China, and then compares its differences and strengths to the existing 2SFCA (two-step floating catchment area) methods. The experimental results demonstrate that the proposed method effectively quantifies the actual fire service needs and reflects a more realistic spatial pattern of accessibility (i.e., high accessibility level corresponded to a low fire service needs). In addition, we teste the relationship between service accessibility and the facility busyness using the inverted 2SFCA method. The empirical findings indicate that the weighted average accessibility obtained by F-2SFCA is reciprocal to facility busyness across the study area (based on a 5-min catchment), and fits an obvious nonlinear correlation with the high R-square values. The above results further prove the effectiveness and accuracy of the proposed method in characterizing the accessibility of fire services.
\end{abstract}

Keywords: fire facility; non-spatial effects; fire service accessibility; 2SFCA; facility busyness

\section{Introduction}

Fire stations, as important public service facilities that provide professional fire services, came into existence with the aim of fighting fires, natural disasters, and other local hazards [1]. Nowadays, there exist many problems in the utilization of fire facilities in China, such as the dispersal of fire stations and fire hydrants, larger areas of responsibility, and inefficient service capabilities. Therefore, the rationality for the spatial distribution of fire facilities affects their allocation fairness and efficiency, and the quantity and quality of public services. To guarantee adequate access to fire services, reasonable planning and allocation of fire facilities require the accurate measurement of accessibility to the actual service demand locations.

Good access to public services that provide adequate resources or facilities is widely regarded as an important indicator for the quality of urban life, such as healthcare, green space, transit stations, and other basic amenities. For fire emergency services, high accessibility levels help improve the efficiency of operational response, and reduce injuries and deaths. Accessibility is often defined as the ease and comfort with which a person reaches a facility or an activity (e.g., health care, food stores) from a particular location [2,3]. Generally, accessibility involves two elements: (i) regional availability and (ii) regional 
proximity [2]. The regional availability can be considered as the ratio of supply-demand for each demand location in a given threshold. The regional proximity accounts for the spatial interactions between supply and demand sides. The gravity model is the combination of availability and accessibility [4,5], although it is reasonable in theory, it involves much computation and the result is not intuitive to interpret [6]. To handle the above limitations, the floating catchment area (FCA) method was put forward, which incorporated three critical factors in measuring accessibility level: potential demand, supply, and geographic impedance [6]. Unlike traditional gravity-based model, the FCA method defines catchment areas by measuring travel cost on a continuous scale using floating catchment areas instead of set boundaries. Thus, the FCA method can be utilized to account for spatial variability in demand and supply for services across areas. Building on original version of FCA, various modified versions of FCA metrics have been developed to measure accessibility [7-10]. Among these extensions, the two-step floating catchment area method (2SFCA) is the most frequently used in numerous research and applications. The 2SFCA is a special case of the gravity model, which is more intuitive and easier to interpret and calculate than the original gravity model. A major advancement in the 2SFCA method is the use of catchments that are specific to population and service locations rather than based on the predefined districts [11,12]. As a result of the above advantages, the 2SFCA model has been extensively applied to the spatial accessibility analysis in a number of applications $[7,8,13,14]$. Furthermore, in order to obtain more reliable measures of the accessibility to services, many improvements of the 2SFCA models have been developed. For example, McGrail and Humphreys introduced a capped access threshold to define the catchment size based on different weights in the whole city areas [7,8]. Luo and Whippo (2012) proposed a variable two-step floating catchment area method to dynamically determine catchment sizes by gradually increasing the catchment until a base physician-to-population ratio was met [15]. Given truly optimal configurations are highly unlikely in real cases, a modified two-step floating catchment area (M2SFCA) metric was presented to address this issue [16]. Recently, Kocatepe et al. (2017) utilized an empirical-Gaussian two-step floating catchment area (EG-2SFCA) method to investigate the exposure of different population groups to severe injury crash hotspots based on roadway network distances and a socioeconomic-based weighting approach [17].

Numerous studies have used different types of accessibility measures to estimate access to healthcare or other public services, however, little if any consideration has been given to the accessibility of urban emergency services. Fire service access may be considered as the interface between potential fire hazard locations and fire prevention resources, and is affected by characteristics of both those who provide and those who utilize the services. With regard to the supply side, quick access to the fire scene and the implementation of timely and effective fire services are critical to reducing the loss of lives and properties. From the demand side, high-risk fire regions require more services than areas where they rarely happen. The assessment of spatial access to fire service involves accounting for spatial interaction, supply, and demand, and is influenced by spatial and non-spatial effects. With respect to the spatial aspect, access to fire services may be affected by a series of factors, including the spatial distribution of fire facilities, time, or distance impedance to available services. Due to the special characteristics of fire services, the inappropriate allocation of prevention resources and unreasonable distance impedance may hinder the effective implementation of immediate services. For the non-spatial perspective, fire service needs and facility capacity are two important elements in assessing the supply-demand relationship of spatial accessibility. Fire service needs reflect a residential area's potential fire risk state (e.g., fire occurrence frequency and fire-prone spots), as places where fires often occur demand more fire services. Conventional measures are based on population size or density to estimate service needs, however, these approaches may overlook the characteristics and complexity of emergency services and result in misleading estimates. The service quality of the fire station depends on its specialization, response experience, and number of firefighting appliances serving in it. However, the accurate measurement is complex and difficult. For simplicity, in this study fire facility capacity is classified according to the number of fire fighters and fire trucks serving in them. Usually, larger facility capacity, smaller population size, and weaker distance impedance indicate 
higher spatial access to facility services [14]. Besides, facility busyness (stress level or crowdedness) is an important non-spatial measurement to reflect the supply-to-demand ratio [18]. In this paper, facility busyness is used to measure the degree of congestion for a service provided at a fire station.

In this article, an improved 2SFCA based on a fire service (F-2SFCA) approach is proposed by incorporating both spatial and non-spatial factors in assessing the spatial accessibility of fire services. Specifically, it incorporates a service competition model to solve the impact of both spatial impedance (distance or time) and facility capacity on spatial accessibility [19], and quantifies the demand for fire services by establishing a weighted sum coefficient based on population size, fire frequency, and floor area. Then, it compares and analyzes the differences in the results calculated by the original 2SFCA, the 2SFCA with spatial factors, and the proposed F-2SFCA models in the case study of access to urban fire services. Finally, it utilizes the facility busyness effect of i2SFCA (inverted two-step floating catchment area) to explain why the proposed F-2SFCA method tends to show a higher accessibility score in residential locations corresponding to a lower busyness in fire facilities there, as well as to further verify its rationality to measure accessibility for urban fire service [18].

\section{Review of Key Improvements for the Floating Catchment Area Methods}

\subsection{Improvement 1: Addition of Distance Decay Function}

Choosing an appropriate distance decay function is the primary issue in building a spatial accessibility model. Several studies have created distance-decay functions in some cases [20], among them, the three most frequently used impedance functions include continuous function (e.g., gravity-type function), discrete step-wise function, or a hybrid function form of the previous two. However, there is little empirical evidence to guide the appropriate functional form for the distance decay weights. In practical application, the selection of distance decay function forms should be based on the actual travel behaviors in the case studied. In this study, we use the kernel density weight function to explain the distance decay. The kernel density type function belongs to a concave function; when the distance is smaller, the decay rate of accessibility with distance is slower, whereas the greater the distance, the faster the decay [21]. The concrete formulation can be written as

$$
g\left(d_{i j}\right)=\frac{3}{4}\left[1-\left(\frac{d_{i j}}{d_{0}}\right)^{2}\right], d_{i j} \leq d_{0}
$$

where $g\left(d_{i j}\right)$ is the distance impedance based on the kernel density function, $d_{i j}$ is the travel distance from $i$ to $j$, and $d_{0}$ is the threshold travel distance.

\subsection{Improvement 2: Addition of Service Competition Model}

The second issue is with respect to the potential for competition among multiple facilities. The conventional version of 2SFCA models are deficient in illuminating the resource allocation effect, given their inappropriate assumption of population demand, and therefore may lead to overestimation for some facilities in some cases. To handle this limitation, Wan et al. (2012) proposed a three-step floating catchment area method (3SFCA) to effectively resolve the inaccurate estimation of demand in the E2SFCA method [14]. The primary contribution of the 3SFCA method over previous models lies in its more reasonable assumption of the competition of multiple facilities. Moreover, the competition among service facilities is attached to the original E2SFCA model.

However, the competition weight of the 3SFCA does not consider the supply side effect on peoples' choice. Therefore, it is necessary to alter the competition weight by adjusting the facility capacity differences between the available facilities. Aiming to address the overestimation or underestimation of population demand, a modified method was proposed by Luo J (2014), which integrated the Huff Model and the floating catchment area method, and the improvement will be helpful for facility resource planning and allocation [19]. 


\subsection{Improvement 3: Addition of Non-Spatial Factors}

Public facility service access is determined by multiple factors and needs to account for both spatial and non-spatial dimensions simultaneously [22,23]. In this paper, residential service demand and facility capacity are considered as important non-spatial factors to emphasize the connection between non-spatial effect and service access. Due to the complexity of calculating the service needs, it is difficult to distinguish the actual needs for fire services within each basic statistical unit [24]. If a region has a low fire incidence rate, then the relative service demand within that population is lower than denoted by only the raw population size [25]. In other words, high fire risk regions should be assigned larger weights to residential areas with high service needs. For the accurate estimation of fire service needs, in this study the fire frequency effect is integrated to quantify the service demand.

Fire facility busyness refers to the ratio between the demand amount of the service and fire facility supply capacity. Recently, Wang (2017) introduced the inverted 2SFCA (i2SFCA) method to assess potential busyness for facilities in areas where inhabitants had better access to healthcare, meanwhile those hospitals in the same areas were often less crowded [18]. In other words, a higher accessibility score in residential areas corresponded to a lower busyness in facilities nearby. By converting the supply and demand forms in the original 2SFCA method, it utilized the i2SFCA method to measure the "busyness" for a service facility. By comparison formulations, we conclude that the average accessibility derived by 2SFCA and the busyness derived by i2SFCA are the reciprocal of each other (see Table 1).

Table 1. Comparison of 2SFCA (two-step floating catchment area) and i2SFCA (inverted two-step floating catchment area).

\begin{tabular}{ccc}
\hline & 2SFCA & i2SFCA \\
\hline Purpose & Measuring service accessibility at a demand location $(i)$ & Measuring busyness at a supply location $(j)$ \\
Ctep 1 & Calculate the supply-demand ratio $R_{j}:$ & $r_{i}=D_{i} / \sum_{l=1}^{n}\left[S_{l} g\left(d_{i l}\right)\right]$ \\
& $R_{j}=S_{j} / \sum_{k=1}^{m}\left[D_{k} g\left(d_{k j}\right)\right]$ & Acquire the facility busyness $C_{j}:$ \\
Step 2 & $A_{i}=\sum_{j=1}^{n}\left[R_{j} g\left(d_{i j}\right)\right]=\sum_{j=1}^{n}\left[S_{j} g\left(d_{i j}\right) / \sum_{k=1}^{m}\left(D_{k} g\left(d_{k j}\right)\right)\right]$ & $C_{j}=\sum_{i=1}^{m}\left[r_{i} g\left(d_{i j}\right)\right]=\sum_{i=1}^{m}\left[D_{i} g\left(d_{i j}\right) / \sum_{l=1}^{n}\left(S_{l} g\left(d_{i l}\right)\right)\right]$ \\
Conclusion & Average accessibility and busyness are reciprocal of each other \\
\hline
\end{tabular}

\section{The Proposed F-2SFCA Method for Fire Service}

Understanding fire service access requires considering both the spatial and non-spatial factors and their interactions. With respect to the spatial dimension, access to services is determined by where the fire facilities are distributed and their supply capacity, and the distance or time impedance from the fire services to residential location. The competition weight of a residential location on fire services is considered in this study, which represents the probability for residents to access to a service site out of all the available service sites within the catchment. Furthermore, a continuous kernel density function is employed instead of the arbitrarily defined subzones of the previous floating catchment area methods. Regarding the non-spatial perspective, fire service needs reflected the true utilization behavior of the population, because various population groups have different levels of fire service needs. However, the quantization of service demand was simply defined by its service population size in previous studies [12]. Statistics show that more than $70 \%$ of urban fires take place in city-building-dense regions and residential areas due to their relatively higher fire occurrence frequencies. In this paper we particularly focus on the two significant factors assessing urban fire risk: historical fire incidence and the number of casualties [26]. Generally, high fire incidence areas tend to utilize more fire services because of their potential fire risks. To quantify the actual utilization behavior of fire services for each residential area, in this study a fire service needs indicator is used to weigh the demand. Finally, the effect of spatial and non-spatial factors are integrated within the 2SFCA method to estimate access. The spatial and non-spatial factors used in this study are shown in Figure 1. 


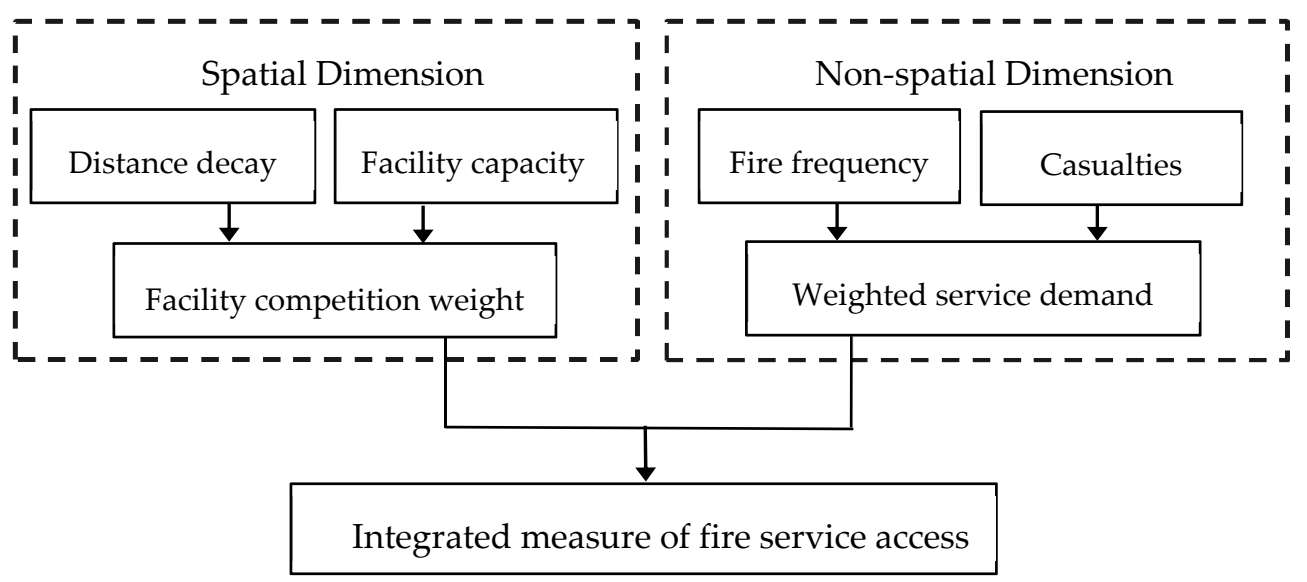

Figure 1. Spatial and non-spatial factors affecting fire service access.

\subsection{Measuring Fire Service Accessibility Integrating Spatial and Non-Spatial Factors}

\subsubsection{Modeling Spatial Effect Utilizing Spatial Interaction Model}

Since fire services are not evenly distributed, spatial impedance between supply and demand locations is deemed to be a key access barrier for fire service. Instead of dividing the catchment area into several subzones, we applied the kernel density distance decay function continuously in each catchment, which avoided the effects of pre-defined subzones. The distance decay function expression is shown in Equation (1).

Analysis of the facility competition scheme is critical to moderate the inappropriate assumption of facility resource allocation [14]. With the aim of overcoming the problem of both over or under estimating population demand, the distance decay function and service facility capacity are integrated to calculate the facility competition weight. Consequently, a competition weight between each fire service location $j$ and residential location $i$ is calculated by Equation (2), which depends on two parameters: distance to the service facility and fire service capacity.

$$
K_{i j}=\frac{S_{j} \times g\left(d_{i j}\right)}{\sum_{h \in\left\{d_{i h} \leq d_{0}\right\}} S_{h} \times g\left(d_{i h}\right)}
$$

where $K_{i j}$ expresses the competition weight between residential location $i$ and fire facility location $j ; d_{i j}$ represents the travel time (measured by shortest-path distance) from residential location $i$ to any fire facility location $j$ within the catchment; $d_{0}$ indicates the maximum threshold distance (10 min in this study); $d_{i h}$ is the travel time between residential location $i$ and the fire facility location $h$ (i.e., $d_{i h} \leq d_{0}$ ); $g(d)$ is the distance impedance function defined in Equation (1), which determines how travel time impacts the accessibility; $S_{j}$ is the supply capacity (here denoted by the product of firefighters and fire-engines) of service facility $j$ and $S_{h}$ is the supply capacity at location $h$ within the catchment (i.e., $\left.d_{i h} \leq d_{0}\right)$.

\subsubsection{Assessing Non-Spatial Effect by Quantifying Service Needs}

Residential areas differ in terms of fire service needs in line with their building structure, population density, neighboring environment, and other non-spatial characteristics [27]. Based on the historical fire incidence and the number of casualties in each residential area $i$, we developed a fire service needs indicator. In this study, the fire service needs in residential location $i$ is measured as the probability of affecting fire service access in the non-spatial dimension, $\beta_{i}$, defined by a weighted sum expression:

$$
\beta_{i}=\left[\frac{F_{i}}{F_{\max }}\right]+\left[\frac{E_{i}}{E_{\max }}\right]
$$


where $F_{i}$ represents the number of fires in residential location $i$ and $F_{\max }$ expresses the largest number of fires in all areas; $E_{i}$ indicates the number of casualties in residential location $i$; and $E_{\max }$ denotes the largest the number of casualties in all areas; the value of $\beta_{i}$ lies between 0 and 2 . This can be understood as the higher the fire frequency and larger casualties in an area, the higher the service demand.

To moderate the overestimation or underestimation of population demand on fire service facilities in the E2SFCA, the weighted sum expression is then added to the calculation of the demand for fire service. That is,

$$
D_{i}=\beta_{i} \times P_{i}
$$

where $D_{i}$ represents the adjusted demand of residential location $i$ for fire service, $P_{i}$ is the population size (number of dwellers) in residential location $i$.

\subsection{Integrating Spatial and Non-Spatial Effects within the 2SFCA Method}

Based on these improvements above, we aim to incorporate spatial and non-spatial factors within the 2SFCA model to improve the accuracy of fire service accessibility analysis; here, we refer to the improved 2SFCA method based on fire services as the F-2SFCA model in this study. The modified steps are implemented as follows:

Inspired by the previous research $[5,6,14,15]$, this article applies multiple travel time zones across a catchment to differentiate accessibility scores. First, we define a 10-min maximum catchment size for each fire facility. Then, we divide each catchment into three travel time zones (0-5 min and 5-10 min) and calculate the supply-demand ratio $R$ for a service facility $j$ by

$$
R_{j}=\frac{S_{j}}{\sum_{r=1,2} \sum_{l \in D_{r}} D_{l} \times K_{l j} \times g\left(d_{l j}\right)}=\frac{S_{j}}{\sum_{l \in D_{1}} D_{l} \times K_{l j} \times g\left(d_{l j}\right)+\sum_{l \in D_{2}} D_{l} \times K_{l j} \times g\left(d_{l j}\right)}
$$

where $D_{l}$ is the adjusted potential demand of residential location $l$ for fire service facility $j$; $D_{r}$ is the $r$-th travel time zone $(r=1,2)$ that falls within the catchment.

Considering the distance impedance within a catchment and facility competition weight, the accessibility score of residential location $i$ can be revised as:

$$
A_{i}=\sum_{r=1,2} \sum_{j \in D_{r}} K_{i j} \times R_{j} \times g\left(d_{i j}\right)=\sum_{j \in D_{1}} K_{i j} \times R_{j} \times g\left(d_{i j}\right)+\sum_{j \in D_{2}} K_{i j} \times R_{j} \times g\left(d_{i j}\right)
$$

where $A_{i}$ represents the accessibility of residential location $i$ to fire services.

\subsection{Measuring Facility Busyness}

To achieve the goal of optimizing the allocation of fire facility resources, reasonable service planning requires reliable measures of the potential busyness for fire facilities. This paper utilizes the i2SFCA method to capture the busyness for fire facilities. As mentioned previously, the total amount of service demand served by any facility $j$ within the catchment can be indicated by

$$
V_{j}=\sum_{r=1,2} \sum_{l \in D_{r}} D_{l} \times K_{l j} \times g\left(d_{l j}\right)
$$

Hence, the ratio of total demand to total supply capacity is represented as

$$
C_{j}=V_{j} / S_{j}
$$

where the ratio $C_{j}$ can be considered as the potential busyness of the fire facility (e.g., patients per hospital). The higher value $C_{j}$ demonstrates that a facility tends to be more crowded. 


\section{Case Study}

\subsection{Study Area and Datasets}

Our case study focuses on the central part of Nanjing metropolitan area, China, which covers an area of about $1043 \mathrm{~km}^{2}$, with a total population of more than 5.23 million in 2016 (see Figure 2). In accordance with "The 13th Five-Year Plan for City Fire Planning of Nanjing", published by the Nanjing Fire Department in 2017, the following five types of high-risk fire regions (as depicted in Figure 2c) were confirmed in the urban areas of Nanjing: massive high-rise building concentrated areas, urban commercial concentration zones, underground spaces, chemical industry parks, and urban villages [28]. For these reasons, Nanjing is selected as a typical case study area for fire service access.

In China, residential quarters area the smallest geographic units for neighborhoods where extensive socioeconomic statistics are available [29,30]. Hence, the residential quarter is used as the basic spatial analysis unit in the case study (a total of 2203, shown in Figure 2a). In addition, to obtain more accurate results, the entrance locations (point vector data) of each residential quarter (based on residential boundary data) are used to represent the centroid of population distribution. Our case study involved three categories of datasets: (1) Administrative boundaries of the study area, road network, and other geographical features (e.g., the terrains, the Yangtze River, and the lakes) were provided by the Nanjing Bureau of Surveying and Mapping; (2) demographic data for the residential quarter (residential locations distribution and population size) were collected from the Real Estate Information Center of Nanjing; (3) fire incident records (a total of 1428 fire cases) for 2015-2016 (e.g., fire source location, address, arrival time, burned area, fatality, or injury, etc.) and fire facility conditions (e.g., the number of firefighters, fire engines, and extinguishing equipment) were obtained from the Jiangsu Province Fire Department.

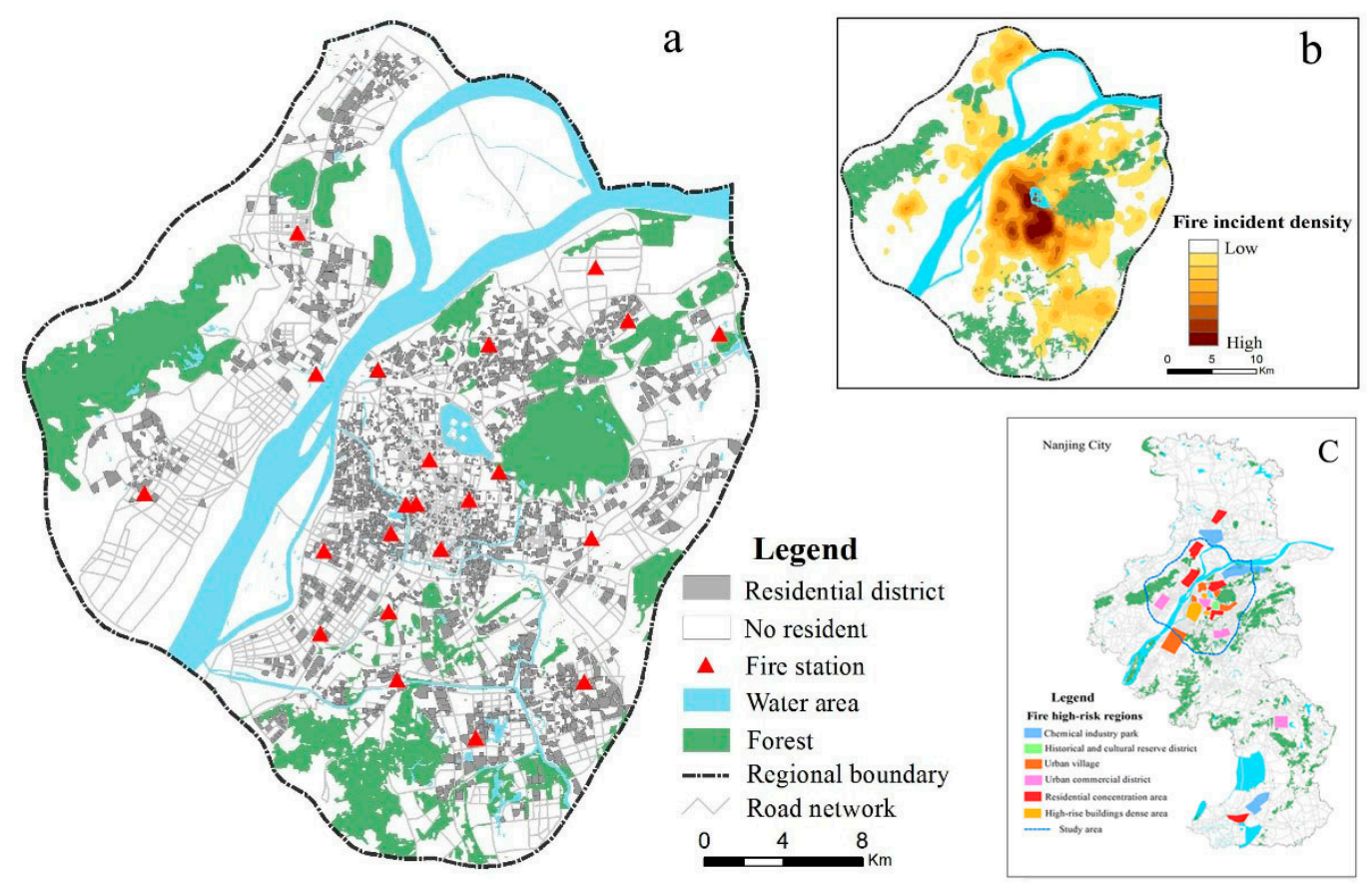

Figure 2. Descriptions of the study area: (a) Study area and basic datasets. (b) Fire density distribution. (c) High-risk fire regions of Nanjing city.

Travel time was estimated as the shortest time through the road networks between any pair of resident locations and fire stations, which can be calculated using the Origin-Destination cost matrix function of the ArcGIS Network Analyst Tool. Considering that the travel time between two 
locations in an urban network depends on local street speed, thus we use speed limit to differentiate the traffic conditions.

Regulations of the Ministry of Public Security (GNJ 1-82) [31]: The primary principle of urban fire station distribution is that firefighters can reach the farthest point of its coverage area in $5 \mathrm{~min}$ after they get the fire alarm. Construction Standard of City Fire Station (JB 152-2017) [32]: the "15 min fire time" can be divided into several steps: discover fire ( $4 \mathrm{~min})$, call alarm $(2.5 \mathrm{~min})$, receive alarm $(1 \mathrm{~min})$, rush to the spot $(4 \mathrm{~min}$ ), and extinguish fire $(3.5 \mathrm{~min}$ ). Hence, the optimal response time (from receiving alarm to reaching the fire scene) for fire service is within $5 \mathrm{~min}$. Despite the fire department regulation of a 5 min travel time, the fact is that fire vehicles driving on urban roads will be affected by actual road conditions (e.g., speed limits, one-way roads, and traffic signals) that may result in response time delays. In addition, choosing an appropriate catchment size is very important because it determines whether a service location is accessible. Considering the above factors, in this paper two thresholds ranging from 0 to $10 \mathrm{~min}$ with a $5 \mathrm{~min}$ increment have been mapped (see Figure 3). Catchment sizes less than 5 min will result in zero accessibility in many residential locations, making it difficult to exhibit the fire service accessibility differences. The 5-min increment is selected to examine the variation in accessibility resulting from different thresholds, which is in line with previous studies [11,33].

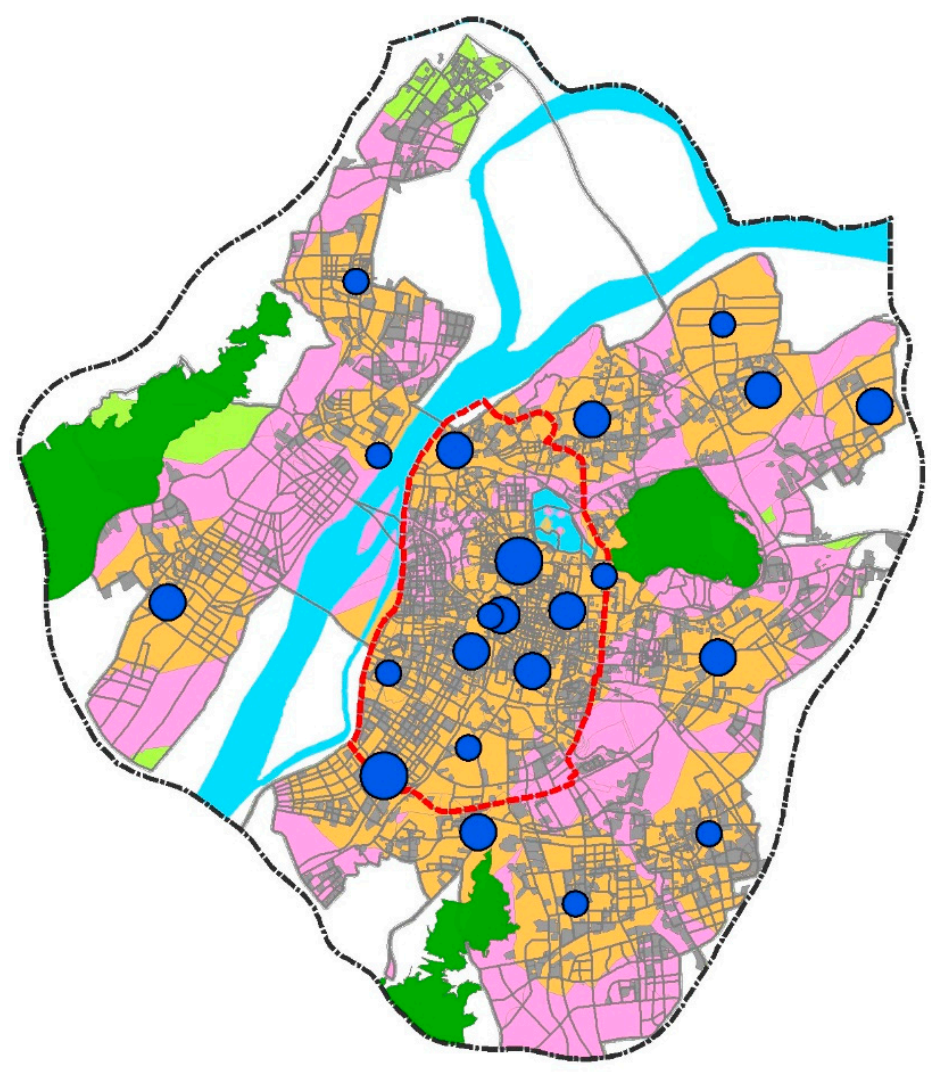

\section{Legend}

Fire Facility Capacity

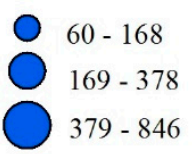

Arrival time[minutes]

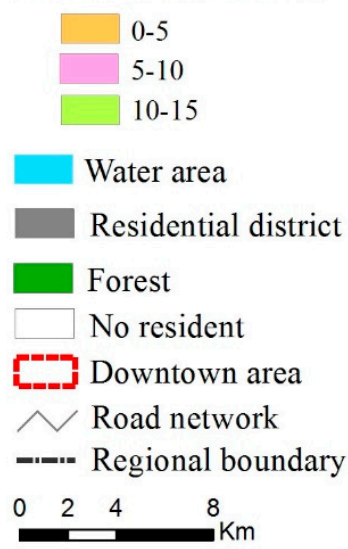

Figure 3. Spatial difference of fire service time accessibility in the study area.

\subsection{Experimental Results and Analysis}

In our accessibility modeling for fire services, three methods are compared, including the original 2SFCA model, the 2SFCA incorporating additional spatial factors (e.g., continuous distance decay function, facility capacity, and facility competition weight), as well as the proposed F-2SFCA method. In order to find out the differences between the different models, quartile classification is employed to sort out the accessibility scores by descending order. The first $25 \%$ of the sorted accessibility scores are assigned a high level, the next $25 \%-50 \%, 50 \%-75 \%$, and the last $25 \%$ are assigned medium, low, and very low levels, respectively. Specifically, comparisons are made according to the relative position 
of the residential quarters' accessibility scores in the three pre-defined districts (areas located in fire high-risk regions). The list of the districts selected for the study with the fire service characteristics are shown in Table 2.

Table 2. Details of districts selected for case study.

\begin{tabular}{ccccccc}
\hline District & Population & F1 & F2 & F3 & F4 & F5 \\
\hline Region I & 391,548 & 84 & 1 & 2 & $2.60 \mathrm{~km}$ & 215 \\
Region II & 399,867 & 96 & 2 & 5 & $2.11 \mathrm{~km}$ & 338 \\
Region III & 296,376 & 42 & 0 & 1 & $2.45 \mathrm{~km}$ & 463 \\
\hline
\end{tabular}

Note: F1: the number of fires, F2: the number of fatalities, F3: the number of casualties, F4: the average travel distance from residential quarter to the nearest fire station, F5: demand/supply ratio (the population size of residential quarters with fires to total facility capacity).

Figure 4 presents the accessibility scores calculated by the original 2SFCA model. Referring back to Figure 2, it can be seen that accessibility scores are high in populous urban centers where fire facility resources are concentrated, and are relatively low in sparsely populated suburbs. The original 2SFCA method demonstrates the resulting pattern of high accessibility in residential quarters within the service catchment and low or very low accessibility outside the catchment. Figure 5 shows the accessibility result map after considering spatial factors, which seems to reveal a more reasonable spatial distribution of accessibility scores when compared to Figure 4 (without considering distance impedance), since incorporating the distance decay function can effectively account for the travel impedance effect within each service catchment throughout the study area [34]. As presented in Figure 5, the highest accessibility is predominantly concentrated in the three urban areas: the central, southern, and northeastern sections. The above areas owned more available fire prevention resources and a perfect transportation network, which means that people in the urban core districts spend less time obtaining fire services than those in the suburbs. In contrast, the southern and northeastern parts of the urban areas had the second-highest accessibility scores, like the Hexi and Xianlin regions. These are newly-developed districts of Nanjing, where the spatial distributions of the residential quarters in Nanjing are extremely even and the infrastructure is adequate to adapt the higher housing occupancy rate. Figure 6 displays the results of applying our proposed F-2SFCA method to measure fire service accessibility, which exhibits a different accessibility pattern due to the integration of spatial and non-spatial factors. As expected, the high accessibility scores are generally nearby for fire service sites considering both the distance impedance and facility capacity such as regions I and II in Figure 6. Compared with Figure 5, several detailed changes in accessibility scores from Figure 6 are noteworthy; the major divergence occurs in the north and south of the downtown district and presents a large discrepancy between the accessibility scores (that is, the north part appeared as medium accessibility, while the south part assumed a high level). This can be explained by the additional non-spatial factor effect, showing that more fire service needs are located in the north, resulting in a relatively low accessibility compared with the south [15]. It can be concluded that although urban center regions are usually abundant in public resources and have short travel distances, there is a highly aggregated population, frequent fire accidents, and high service needs [35], which ultimately may result in the shortage of fire service resources. 


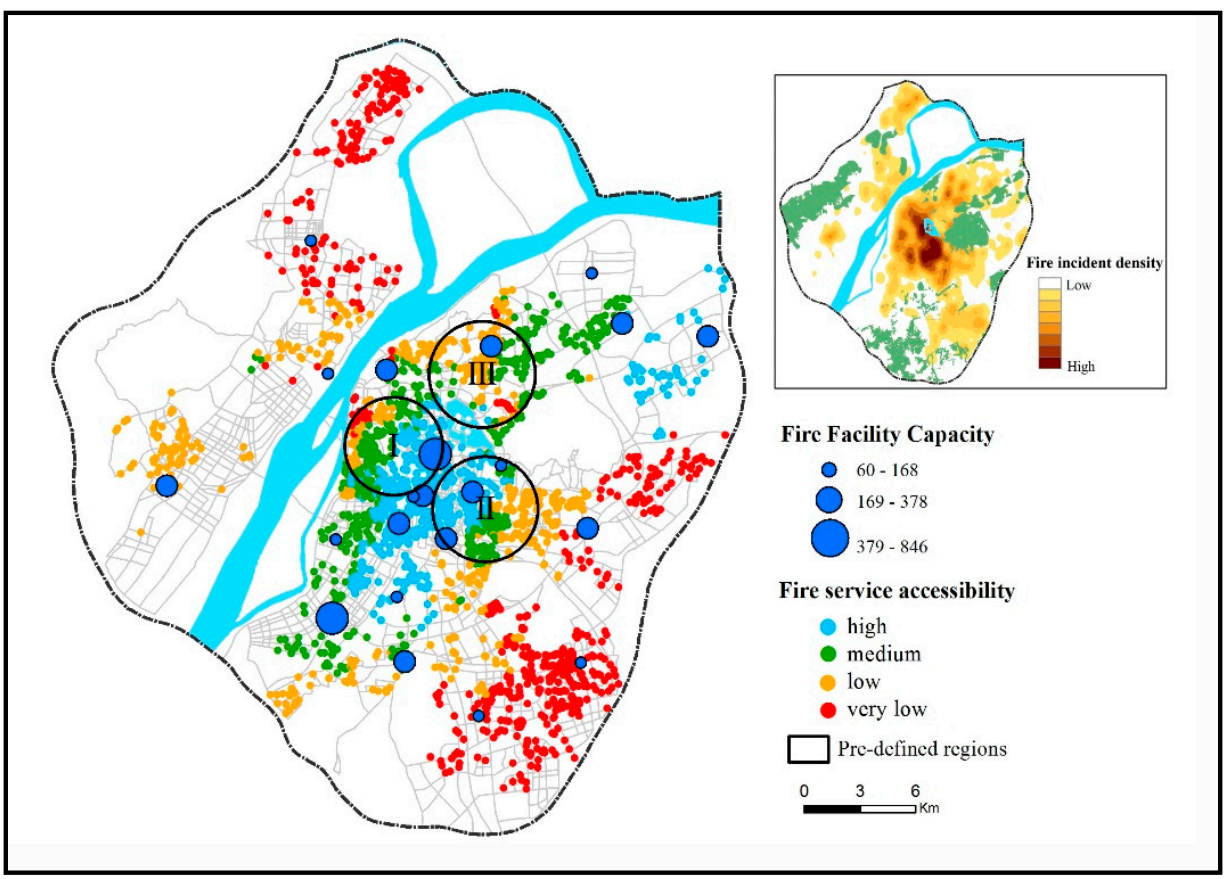

Figure 4. Spatial distribution of accessibility scores using the original 2SFCA method.

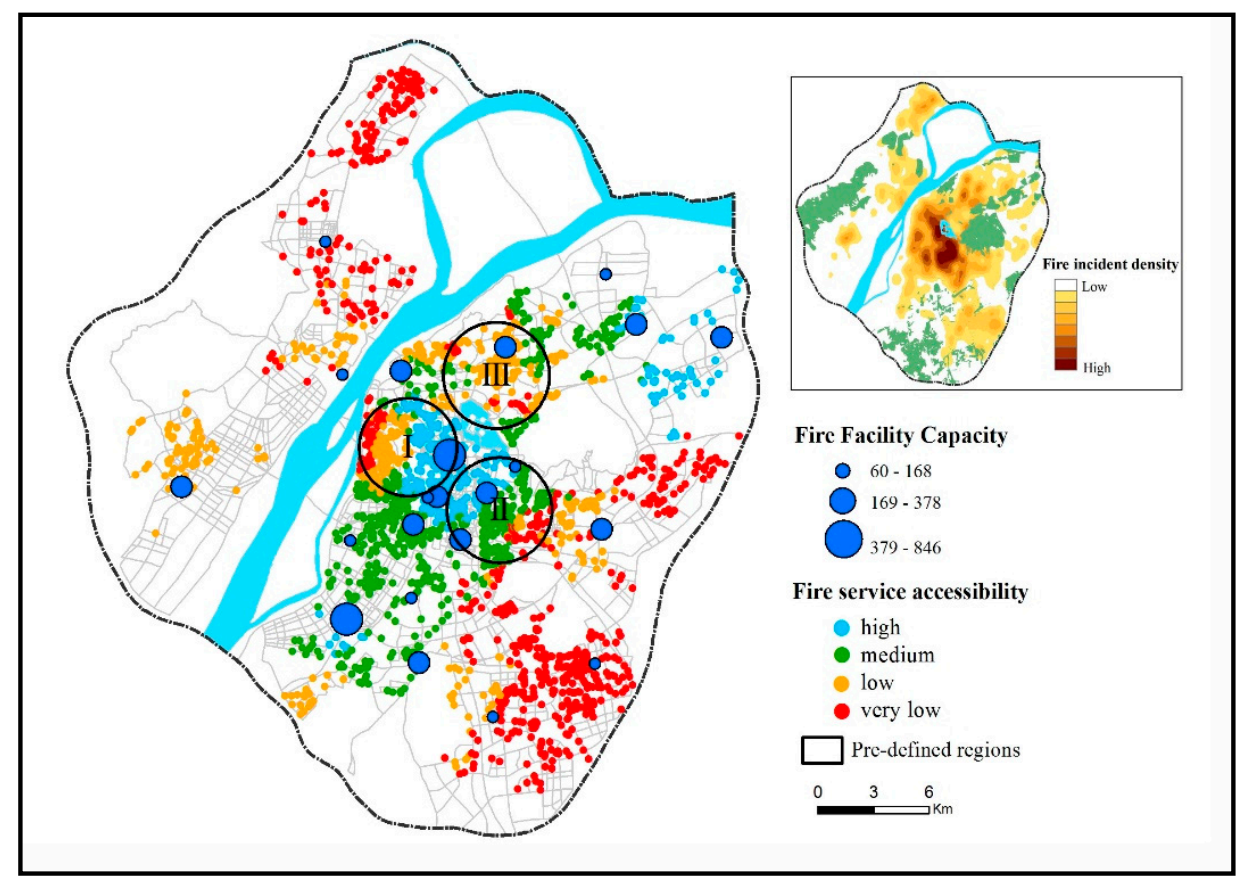

Figure 5. Spatial distribution of accessibility scores using the 2SFCA method incorporating additional spatial factors. 


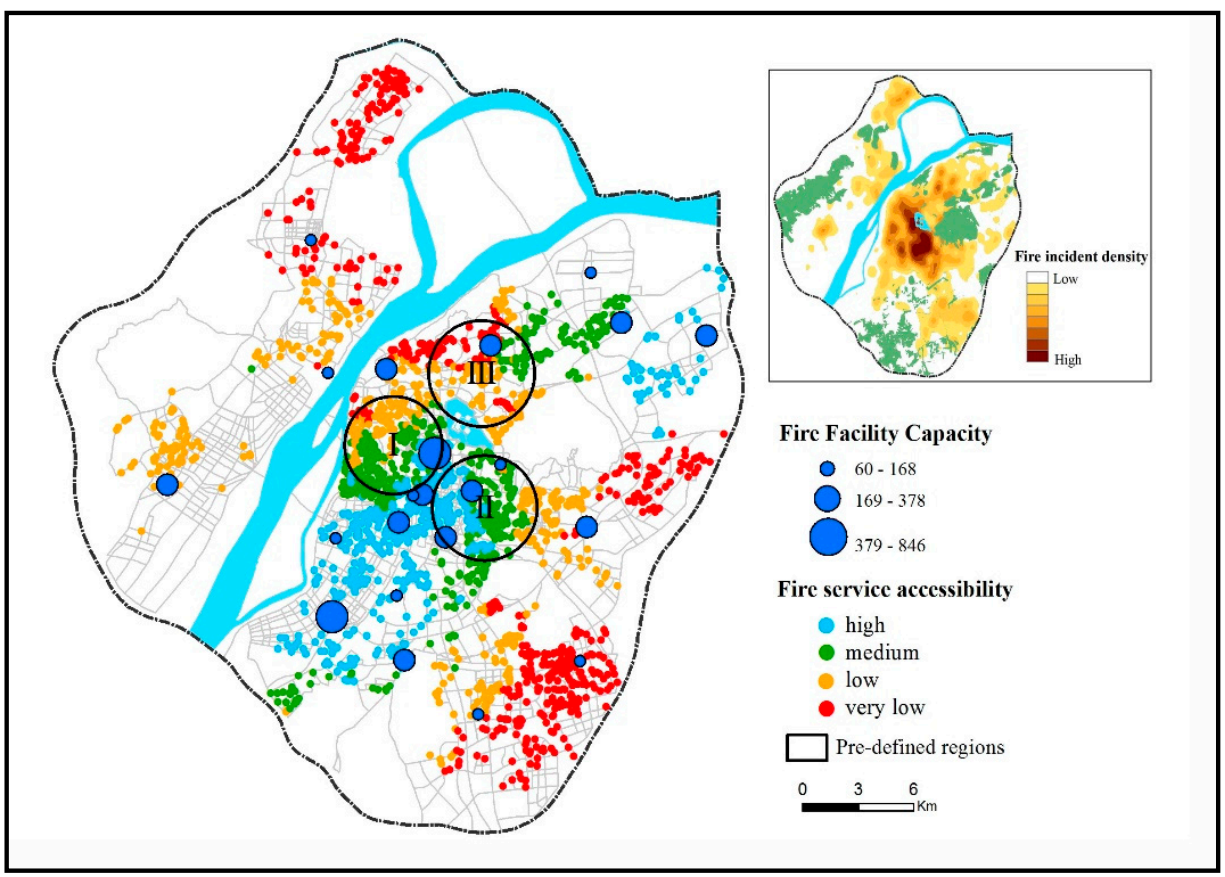

Figure 6. Spatial distribution of accessibility scores using the proposed F-2SFCA method.

For a better understanding of the differences between the different methods, we count a list of fire service parameters that could affect fire service access. As presented in Table 3, within the three groupings (region I, region II, and region III), there is a consistent pattern between decreasing travel distance and increasing accessibility scores due to the incorporation of distance decay function. Clearly, the geographical proximity to fire services has a great effect on accessibility levels. As discussed previously, fire service need reflects a weighting factor for the demand side of fire services. However, the actual fire service demand will be affected by factors such as service population size, fire incidence, and fire facility capacity. To emphasize the importance of non-spatial factors in measuring the accessibility score, fire frequency and demand-supply ratio are two indices used to compare the results obtained by the existing models and our proposed model (see Table 3). Hence, a high fire frequency or demand-supply ratio represents a high demand for fire services. In region I, areas with high fire frequency and high demand-supply ratio (52\% for 81 and $64 \%$ for 107 ) are categorized into the high accessibility level using the original 2SFCA and the 2SFCA method incorporating additional spatial factors. Whereas areas with high accessibility tend to keep a low fire frequency and demand-supply ratio $(14.1 \%, 20)$, as assessed by the proposed method, this means that a low fire service need reduces a facility's catchment size, and thus leads to high access. As illustrated in region II, residential quarters are usually rich in fire resources and have short distances. Compared with the first two methods, the proposed F-2SFCA method decreases the proportion of residential quarters with high fire service need in high accessibility areas $(45 \%)$. Because the proposed model can capture the effect of demand-to-supply ratio change on accessibility scores. For region III, the results reveals that as the accessibility level decreases, the changes of demand-supply ratio tends to increase accordingly. In this table, the accessibility results using the F-2SFCA method show a significant difference in the distribution of fire service access. That is, high fire service needs increase the population size competing for access to available fire resources. Therefore, the residential quarters with high accessibility scores correspond to low fire service needs. The above results reveal that our proposed model focuses on other non-spatial factors in addition to spatial factors, and yields an integrated effect for fire service accessibility assessment. 
Table 3. The parameters describing fire service difference changes calculated by different methods in three pre-defined regions.

\begin{tabular}{|c|c|c|c|c|c|c|c|c|c|}
\hline \multirow{2}{*}{$\begin{array}{l}\text { Accessibility } \\
\text { Level }\end{array}$} & \multicolumn{3}{|c|}{ The Original 2SFCA } & \multicolumn{3}{|c|}{$\begin{array}{l}\text { The 2SFCA Incorporating } \\
\text { Additional Spatial Factors }\end{array}$} & \multicolumn{3}{|c|}{ The F-2SFCA } \\
\hline & F4 & F1 \% & F5 & F4 & F1 \% & F5 & F4 & F1 \% & F5 \\
\hline \multicolumn{10}{|c|}{ Region I } \\
\hline high & $1.50 \mathrm{~km}$ & $52 \%$ & 81 & $1.7 \mathrm{~km}$ & $64 \%$ & 107 & $1.03 \mathrm{~km}$ & $14.1 \%$ & 20 \\
\hline medium & $3.18 \mathrm{~km}$ & $34 \%$ & 80 & $2.9 \mathrm{~km}$ & $6.5 \%$ & 6 & $2.65 \mathrm{~km}$ & $32 \%$ & 107 \\
\hline low & $4.23 \mathrm{~km}$ & $10.3 \%$ & 25 & $3.56 \mathrm{~km}$ & $16.7 \%$ & 50 & $3.37 \mathrm{~km}$ & $52 \%$ & 53 \\
\hline very low & $3.61 \mathrm{~km}$ & $3.7 \%$ & 29 & $4.38 \mathrm{~km}$ & $12.8 \%$ & 52 & $3.52 \mathrm{~km}$ & $1.9 \%$ & 35 \\
\hline \multicolumn{10}{|c|}{ Region II } \\
\hline high & $1.33 \mathrm{~km}$ & $60 \%$ & 142 & $1.1 \mathrm{~km}$ & $64.7 \%$ & 159 & $1.65 \mathrm{~km}$ & $45 \%$ & 126 \\
\hline medium & $2.21 \mathrm{~km}$ & $29.2 \%$ & 157 & $2.6 \mathrm{~km}$ & $30.6 \%$ & 132 & $2.18 \mathrm{~km}$ & $28.8 \%$ & 172 \\
\hline low & $3.32 \mathrm{~km}$ & $10.8 \%$ & 39 & $3.74 \mathrm{~km}$ & $4.7 \%$ & 47 & $3.53 \mathrm{~km}$ & $26.2 \%$ & 40 \\
\hline very low & - & - & - & - & - & - & - & - & - \\
\hline \multicolumn{10}{|c|}{ Region III } \\
\hline high & - & - & - & - & - & - & - & & - \\
\hline medium & $2.53 \mathrm{~km}$ & $43 \%$ & 252 & $2.56 \mathrm{~km}$ & $42 \%$ & 247 & $2.49 \mathrm{~km}$ & $24.5 \%$ & 77 \\
\hline low & $2.92 \mathrm{~km}$ & $50 \%$ & 196 & $2.19 \mathrm{~km}$ & $38 \%$ & 182 & $2.12 \mathrm{~km}$ & $42 \%$ & 241 \\
\hline very low & $4.11 \mathrm{~km}$ & $7 \%$ & 15 & $3.75 \mathrm{~km}$ & $20 \%$ & 34 & $2.67 \mathrm{~km}$ & $33.5 \%$ & 145 \\
\hline
\end{tabular}

Note: F4: the average travel distance from residential quarter to the nearest fire station. F1: the number of fires. F5: demand/supply ratio (the population size of residential quarters with fires to total facility capacity).

\subsection{Evaluating the Performance of F-2SFCA by Inverted 2SFCA}

Travel distance, service needs, and fire facility capacity are key features in assessing fire service accessibility. The 2SFCA method has been broadly used as an effective approach to estimate spatial accessibility. Recently, an i2SFCA method was proposed by Wang (2017), which revealed that a higher accessibility value in residential quarters was closely related to a lower busyness in facilities there [14]. Motivated by rational derivation, in this paper, we aimed to examine the relationship between resident-based accessibility and facility-oriented busyness, and further validate the reasonableness of the proposed F-2SFCA method.

Accurate measurements are difficult to implement regarding the busyness of the fire facility. In this case study, the facility busyness is assessed by the ratio of demand amount within the catchment of a service to facility capacity. Figure 7 presents the accessibility scores of the residential quarters and facility busyness values across the whole study area based on a 5-min catchment. The result showed that the average accessibility score in the residential quarters around each fire station dropped as the fire facility busyness increased. The corresponding relationship between the accessibility scores and facility busyness fits the logarithm function quite well with the high R-squared value (0.61), as presented in Figure 8. In addition, the average congestion index in the study area is 76 , which is exactly equal to the inverse of the weighted average accessibility (i.e., 1/0.013). The resulting patterns are largely consistent when selecting other distance decay functional forms or adopting different catchment sizes. In conclusion, the analysis results clearly demonstrate that the average accessibility calculated by the F-2SFCA method is the reciprocal of the average facility busyness across the whole study area. 


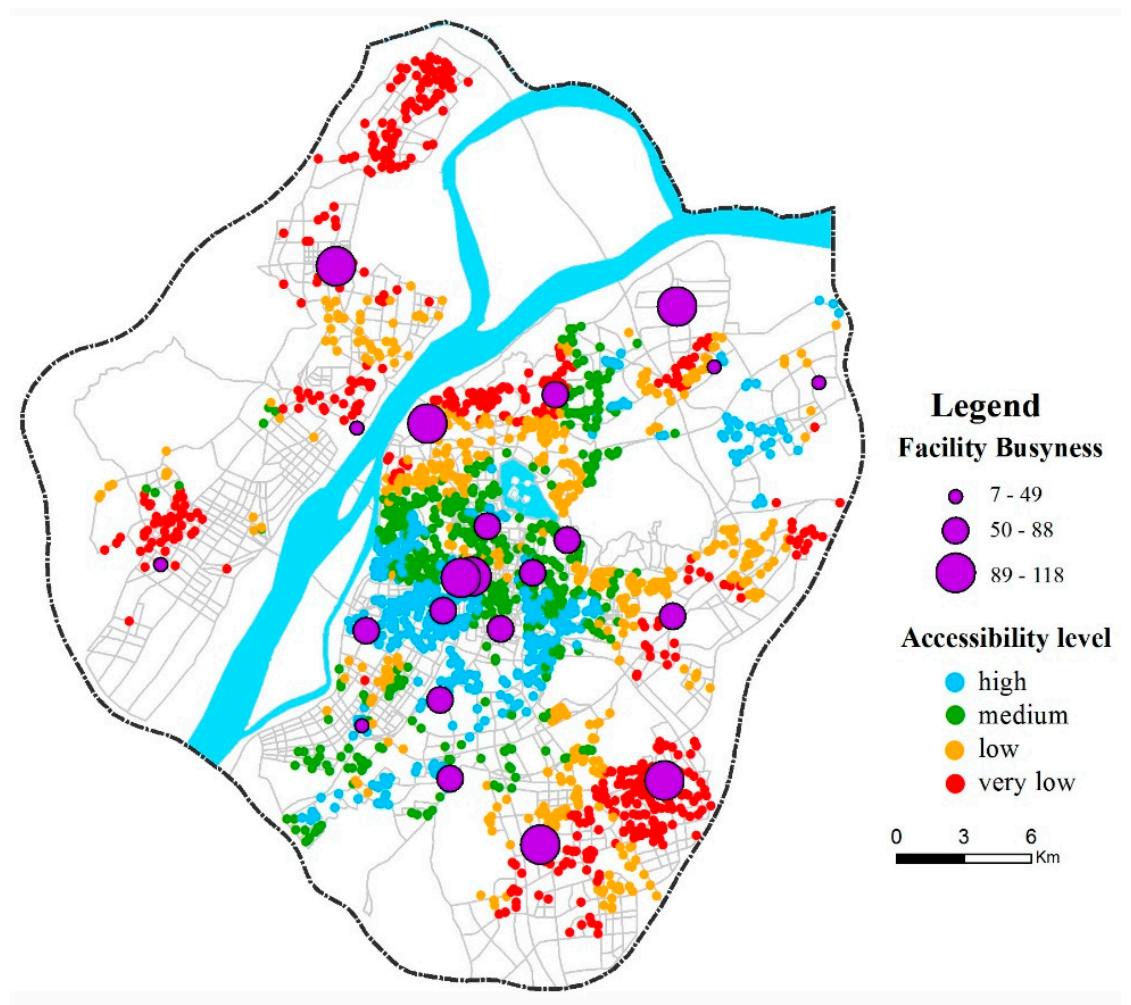

Figure 7. Fire facility busyness and residential quarter accessibility score across the whole study area (based on 5-min catchment).

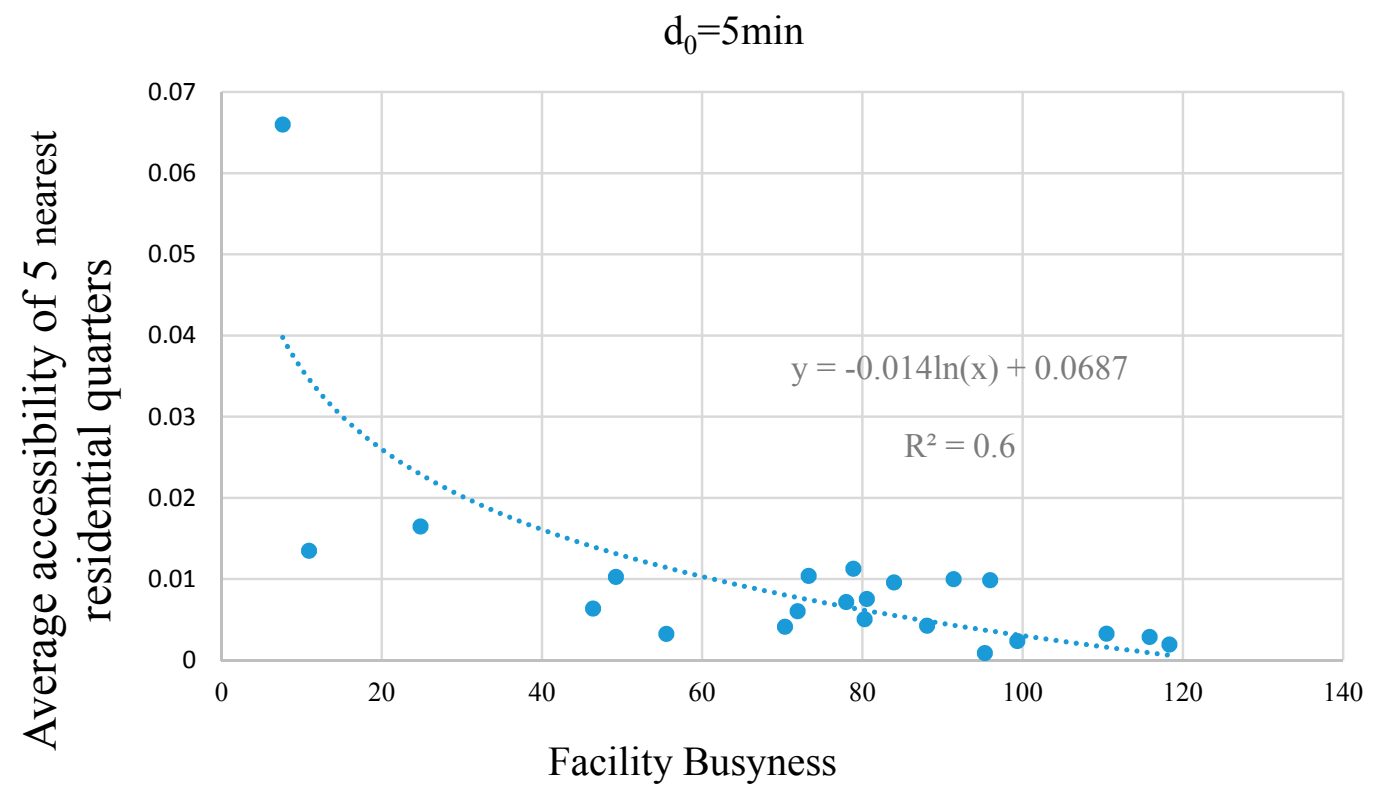

Figure 8. Fire facility busyness versus averaged residential quarter accessibility across the whole study area (based on 5-min travel time).

\section{Conclusions}

Fire service accessibility in residential areas changes with space and is affected by both spatial and non-spatial factors. Thus, in this article, we present an improved F-2SFCA method to measure the access to fire services in urban residential areas. The proposed method drew on a range of factors, including traffic conditions, distance impedance, fire service capacity, fire frequency, and floor area. Our contribution is the combination and adaptation of these factors to produce an integrated model 
of fire service access. The major advantage of the improved F-2SFCA model over previous studies is that our proposed method develop a weighted sum coefficient to adjust the "real" fire service demand behavior in residential areas, which highlights the relationship between the non-spatial dimension and fire access. Moreover, this research adopts the smallest available geographical unit (residential quarters) as census tracts, which enabled our proposed method to measure access at a fine scale. As a result, application of the proposed method is likely to obtain a more realistic distribution of fire service access.

Accordingly, a case study was designed in an urban area of Nanjing, China, to test the sensitivity of the proposed method. When comparing the results of F-2SFCA against the 2SFCA that incorporated spatial factors, the spatial distribution of accessibility scores in suburban areas is almost consistent due to the influence of the distance decay function. However, there is a great difference in the distributions of the accessibility scores in the urban center areas between the above two approaches. To be specific, the 2SFCA method incorporating spatial factors focuses on the distance decay effect and catchment size in characterizing the fire service accessibility, and as a result, high accessibility residential quarters are generally close to the fire facility or within each catchment. The proposed F-2SFCA model is concerned not only with the spatial interactions between fire facilities and residential locations, but with which factors affect the actual fire service demand. Compared with the original 2SFCA model, the major changes in accessibility scores appeared in the downtown districts due to the consideration of fire frequency and floor area. By employing the proposed approach, the findings reflect a more realistic spatial pattern of fire service behavior. That is, a residential area with a high fire incidence will accordingly have an increased demand for fire services. The facility busyness effect inherited from the i2SFCA method reveals that the weighted average accessibility calculated by the 2SFCA and i2SFCA-exported busyness are mutually reciprocal. Based on this theory, the experimental results show that the weighted average crowded in the study area calculated by the F-2SFCA is 76, which is exactly the reciprocal of the aforementioned weighted average accessibility score of 0.013 , and further proves that our proposed F-2SFCA method is feasible and reasonable.

Fire facilities are one of the indispensable utilities of urban areas that offer safety and comfort for inhabitants. Built on previous FCA metrics, this study presents a synthetic methodological framework integrating spatial and non-spatial dimensions for capturing more detailed spatial variation of fire service accessibility. However, there are still limitations with our proposed method. First, due to the lack of available empirical data on "real" fire service demand behavior, our study only utilizes the fire incidence and the number of casualties, two factors that weigh population demand, while in reality there might be many other factors that should be considered. Second, our work in the study is highly case dependent and the results cannot be generalized to other regional areas, as fire service access results are highly sensitive to neighborhood socioeconomic characteristics within a metropolitan context. Future studies may consider these limitations and develop more advanced models to quantify fire service demand.

Author Contributions: Zelong Xia and Wenhao Yu conceived and designed the research. Yuehong Chen helped in date analysis and language correction. Zelong $\mathrm{Xia}$ and Hao Li wrote the paper.

Funding: This research was funded by National Key R \& D Program of China, grant number 2018YFC1508603.

Acknowledgments: This study was supported in part by the Open Research Fund Program of Beijing Key Laboratory of Urban Spatial Information Engineering [No. 2018208], in part by the Fundamental Research Funds for the Central Universities [No. 2019B00314], in part by the National Natural Science Foundation of China [No. 41830110], in part by the National Natural Science Foundation of China [No. 41701440], and by the Natural Science Foundation of Hubei Province [No. 2018CFB513].

Conflicts of Interest: The authors declare no conflict of interest.

\section{References}

1. Wiśniewski, S. The use of network analyst tool and 2SFCA method to assess fire service effectiveness in a city, as exemplified by Łódź. Geomat. Landmanag. Landsc. 2017, 147-158. [CrossRef] 
2. Pill, R. Accessibility and utilization: Geographical perspectives on health care delivery. J. R. Coll. Gen. Practition. 1984, 34, 452.

3. Pooler, J. Measuring geographical accessibility: A review of current approaches and problems in the use of population potentials. Geoforum 1987, 18, 269-289. [CrossRef]

4. Shen, Q. Location characteristics of inner-city neighborhoods and employment accessibility of low-income workers. Environ. Plan. B 1998, 25, 345-365. [CrossRef]

5. Luo, W.; Qi, Y. An enhanced two-step floating catchment area (E2SFCA) method for measuring spatial accessibility to primary care physicians. Health Place 2009, 15, 1100-1107. [CrossRef] [PubMed]

6. Luo, W.; Wang, F.H. Measures of spatial accessibility to health care in a GIS environment: Synthesis and a case study in the Chicago region. Environ. Plan. B 2003, 30, 865-884. [CrossRef]

7. Mcgrail, M.R.; Humphreys, J.S. Measuring spatial accessibility to primary care in rural areas: Improving the effectiveness of the two-step floating catchment area method. Appl. Geogr. 2009, 29, 533-541. [CrossRef]

8. McGrail, M.R.; Humphreys, J.S. A new index of access to primary care services in rural areas. Aust. N. Zeal. J. Public Health 2009, 33, 418-423. [CrossRef]

9. Langford, M.; Higgs, G.; Fry, R. Using floating catchment analysis (FCA) techniques to examine intra-urban variations in accessibility to public transport opportunities: The example of Cardiff, Wales. J. Transp. Geogr. 2012, 25, 1-14. [CrossRef]

10. Dony, C.C.; Delmelle, E.M.; Delmelle, E.C. Re-conceptualizing accessibility to parks in multi-modal cities: A variable-width floating catchment area (VFSCA) method. Landsc. Urban Plan. 2015, 143, 90-99. [CrossRef]

11. Dai, D. Racial/ethnic and socioeconomic disparities in urban green space accessibility: Where to intervene? Landsc. Urban Plan. 2011, 102, 234-244. [CrossRef]

12. Ngui, A.N.; Apparicio, P. Optimizing the two-step floating catchment area method for measuring spatial accessibility to medical clinics in Montreal. BMC Health Serv. Res. 2011, 11, 1-12. [CrossRef] [PubMed]

13. Dai, D.; Wang, F. Geographic disparities in accessibility to food stores in southwest Mississippi. Environ. Plan. B 2011, 38, 659-677. [CrossRef]

14. Wan, N.; Zhou, B.; Sternberg, T. A three-step floating catchment area method for analyzing spatial access to health services. Int. J. Geogr. Inf. Sci. 2012, 26, 1073-1089. [CrossRef]

15. Luo, W.; Whippo, T. Variable catchment sizes for the two-step floating catchment area (2SFCA) method. Health Place 2012, 18, 789-795. [CrossRef] [PubMed]

16. Delamater, P.L. Spatial accessibility in suboptimally configured health care systems: A modified two-step floating catchment area (M2SFCA) metric. Health Place 2013, 24, 30-43. [CrossRef]

17. Kocatepe, A.; Ulak, M.B.; Ozguven, E.E.; Horner, M.W.; Arghandeh, R. Socioeconomic characteristics and crash injury exposure: A case study in Florida using two-step floating catchment area method. Appl. Geogr. 2017, 87, 207-221. [CrossRef]

18. Wang, F.H. Inverted Two-Step Floating catchment area method for measuring facility busyness. Prof. Geogr. 2017, 69, 251-260.

19. Luo, J. Integrating the Huff model and floating catchment area methods to analyze spatial access to healthcare services. Trans. GIS 2014, 18, 436-448. [CrossRef]

20. Kanuganti, S.; Sarkar, A.K.; Singh, A.P. Quantifying accessibility to health care using two-step floating catchment area method (2SFCA): A case study in Rajasthan. Transp. Res. Procedia 2016, 17, 391-399. [CrossRef]

21. Cheng, G.; Zeng, X.; Duan, L.; Lu, X.; Sun, H.; Jiang, T.; Li, Y. Spatial difference analysis for accessibility to high level hospitals based on travel time in Shenzhen, China. Habitat Int. 2016, 53, 485-494. [CrossRef]

22. Khan, A.A. An integrated approach to measuring potential spatial access to health care services. Socio-Econ. Plan. Sci. 1992, 26, 275-287. [CrossRef]

23. Wang, F.H. Measurement, optimization, and impact of health care accessibility: A methodological review. Ann. Assoc. Am. Geogr. 2012, 102, 1104-1112. [CrossRef]

24. Li, H.P.; Wang, Q.F.; Shi, W.; Deng, Z.W.; Wang, H.W. Residential clustering and spatial access to public services in Shanghai. Habitat Int. 2014, 46, 119-129. [CrossRef]

25. Tang, J.H.; Chiu, Y.H.; Chiang, P.H.; Su, M.D.; Chan, T.C. A flow-based statistical model integrating spatial and non-spatial dimensions to measure healthcare access. Health Place 2017, 47, 126-138. [CrossRef] 
26. Zhao, Z.Q.; He, B.J.; Li, L.G.; Wang, H.B.; Darko, A. Profile and concentric zonal analysis of relationships between land use/land cover and land surface temperature: Case study of Shenyang, China. Energy Build. 2017, 155, 282-295. [CrossRef]

27. Jennings, C.R. Social and economic characteristics as determinants of residential fire risk in urban neighborhoods: A review of the literature. Fire Saf. J. 2013, 62, 13-19. [CrossRef]

28. Nanjing News. Government Report: The 13th Five-Year Plan for City Fire Plan of Nanjing. Available online: http:/ / www.nanjing.gov.cn/xxgk/szf.htm (accessed on 3 December 2015).

29. Helling, A.; Sawicki, D.S. Race and residential accessibility to shopping and services. Hous. Policy Debate 2003, 14, 69-101. [CrossRef]

30. Larsen, K.; Gilliland, J. Mapping the evolution of 'food deserts' in a Canadian city: Supermarket accessibility in London, Ontario, 1961-2005. Int J. Health Geogr. 2008, 7, 16. [CrossRef]

31. Standards for the Construction of Urban Fire Stations. Available online: http://119.gov.cn/xiaofang/ bzhgzdt/35172.htm (accessed on 15 July 2016).

32. Fire Protection Law of the People's Republic of China. Available online: http://www.119.gov.cn/xiaofang/ bzfg/17213.htm (accessed on 9 June 2015).

33. Dai, D.J. Black residential segregation, disparities in spatial access to health care facilities, and late-stage breast cancer diagnosis in metropolitan Detroit. Health Place 2010, 16, 1038-1052. [CrossRef]

34. Guagliardo, M.F. Spatial accessibility of primary care: Concepts, methods and challenges. Int. J. Health Geogr. 2004, 3, 3. [CrossRef] [PubMed]

35. Ni, J.H.; Wang, J.J.; Rui, Y.; Qian, T.; Wang, J. An Enhanced Variable Two-Step Floating Catchment Area Method for Measuring Spatial Accessibility to Residential Care Facilities in Nanjing. Int. J. Environ. Res. Public Health 2015, 12, 14490-14504. [CrossRef] [PubMed]

(C) 2019 by the authors. Licensee MDPI, Basel, Switzerland. This article is an open access article distributed under the terms and conditions of the Creative Commons Attribution (CC BY) license (http://creativecommons.org/licenses/by/4.0/). 